\title{
PHASE I MAXIMAL USE PHARMACOKINETIC STUDY OF TIRBANIBULIN OINTMENT $1 \%$ IN SUBJECTS WITH ACTINIC KERATOSIS
}

\section{Regina Yavel, ${ }^{1}$ J. Scott Overcash, ${ }^{2}$ Jay Zhi, ${ }^{3}$ Eva Cutler, ${ }^{3}$ David Cutler, ${ }^{3}$ Jane Fang, ${ }^{3}$}

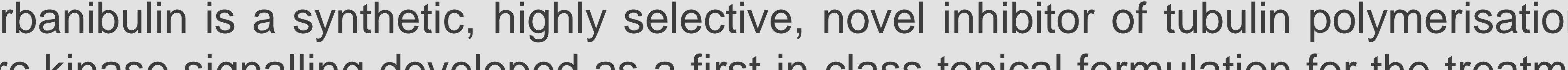
Src kinase signalling
actinic keratosis (AK)

- In Phase I/II studies, tirbanibulin was minimally absorbed and systemic exposure was low when applied topically.

Previous Phase I and II studies showed that tirbanibulin ointment $1 \%$ for 5 days was - Prective against AK lesions on the forearm, face and scalp. Local skin reactions (LSRs) were mostly transient and mild-to-moderate in severity, and tirbanibulin was we the 5-day clinica - Results from two Phase III studies (KX01-AK-003/KX01-AK-004), demonstrated tha (1) 5\%, P<0.0001: KX01-AK-004: 54\% vs. 13\%, P<0.0001) and was well tolented, 5o, $\mathrm{P}<0.001$; KXO1-AK-004: $54 \%$ vs. \#35).

- Here, we present results from a Phase I, open-label, uncontrolled, non-randomised, maximal use pharmacokinetic (PK) study (KX01-AK-007) evaluating the systemic exposure and safety of tirbanibulin ointment $1 \%$ (5 days) applied to the face/balding

\section{OBJECTIVES}

was to determine the PK of tirbanibulin ointment $1 \%$ under maximal use conditions.

Secondary objectives were to evaluate the safety and tolerability of tirbanibulin ointment $1 \%$ and to determine the PK of tirbanibulin metabolites.

\section{METHODS}

Study design

Subjects (aged $\geq 18$ years) with $\geq 6$ clinically typical, visible and discrete AK lesions on $25 \mathrm{~cm}^{2}$ of the face/balding scalp were enrolled in the study.

- Subjects self-applied sufficient tirbanibulin to cover the treatment area $\left(25 \mathrm{~cm}^{2}\right.$ area of he face/balding scalp) from the $250 \mathrm{mg}$ sachet once-daily for 5 consecutive days. 政

Study evaluations

Pharmacokinetics

PK blood sampling (for tirbanibulin and its inactive metabolites [KX2-5036 and KX2

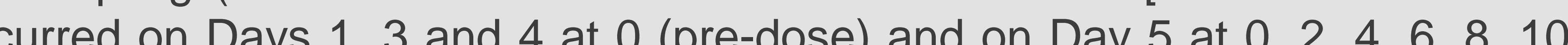
12,16 and 24 hours post-the Day 5 application. Safety

- Adverse events (AEs) were assessed.

- LSRs (erythema, flaking/scaling, crusting, swelling, vesiculation/pustulation, erosion/ulceration; scale of 0-3 [absent-severe]) were evaluated on Days 1, 6, 8, 15 and 29 , and LSR composile scores were calculated as the sum of all individual LSR scores at each visit with the possible range of $0-18$.

\section{RESULTS}

\section{Baseline characteristics}

- In total, 18 subjects (face, $n=9$; scalp, $n=9$ ) were enrolled and completed the study (Table 1). years.

Subjects were White, predominantly male (83.3\%) with Fitzpatrick skin type I-III (94.4\%) and a mean (SD) baseline AK lesion count of 8.2 (2.43 [range: 6-14]). of the full dose possible, $250 \mathrm{mg}$ ).

Table 1. Subject demographics and baseline characteristics

\begin{tabular}{|c|c|c|c|}
\hline Mean Age (SD), years & Face $(n=9)$ & 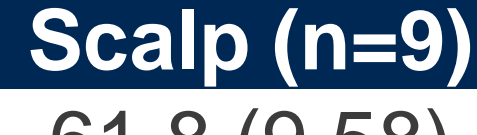 & 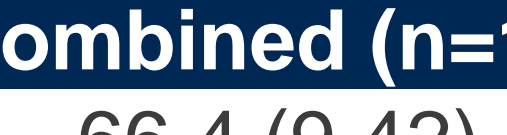 \\
\hline Gender: Female, $\mathrm{n}(\%)$ & $3(33.3)$ & 0 & $3(16.7)$ \\
\hline Male, $n(\%)$ & $6(66.7)$ & $9(100)$ & $15(83.3)$ \\
\hline Race: White, $n$ (\%) & $9(100)$ & $9(100)$ & $18(100)$ \\
\hline \multicolumn{4}{|l|}{ Ethnicity, n (\%) } \\
\hline Hispanic or Latino & 0 & $2(22.2)$ & $2(11.1)$ \\
\hline Not Hispanic or Latino & $9(100)$ & $7(77.8)$ & $16(88.9)$ \\
\hline \multicolumn{4}{|l|}{ Fitzpatrick Skin Type, ${ }^{a}$ (\%) } \\
\hline & $2(22.2)$ & $2(22.2)$ & 4 (22.2) \\
\hline$\|$ & 2 (22.2) & $2(22.2)$ & $4(22.2)$ \\
\hline III & $5(55.6)$ & $4(44.4)$ & $9(50.0)$ \\
\hline IV & 0 & $1(11.1)$ & $1(5.6)$ \\
\hline Mean (SD) Baseline AK lesion count & $8.4(2.46)$ & $7.9(2.52)$ & $8.2(2.43)$ \\
\hline
\end{tabular}

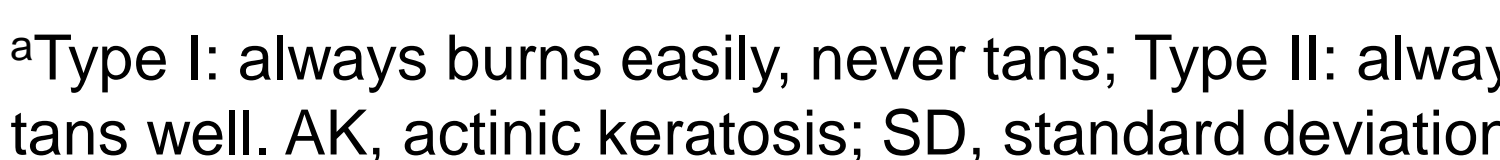

Pharmacokinetics

Tirbanibulin

- Using an LC-MS/MS bioanalytical assay (lower limit of quantification [LLOQ] of 0.01 $\mathrm{ng} / \mathrm{mL}$ ), all subjects had measurable but low concentrations of tirbanibulin at troughs (Figure 1)

- By the observed $\mathrm{C}_{\text {trough }}$ plateau, the pre-dose concentration $\mathrm{C}_{\text {trough }}$ data demonstrated that steady-state was achieved following the third dose (72 hours) of once-daily, 5 days of

dosing.

- On Day 5, mean (SD) $\mathrm{C}_{\max }$ was $0.258(0.231) \mathrm{ng} / \mathrm{mL}(0.598 \mathrm{nM})$, median $\mathrm{t}_{\max }$ was $6.91 \mathrm{~h}$ and mean (SD) AUC $0.24 \mathrm{~h}$ was 4.09 (3.15) $\mathrm{ng} \cdot \mathrm{h} / \mathrm{mL}$ (Table 2).

Tirbanibulin metabolites

- For the majority of subjects, plasma concentrations for the main tirbanibulin metabolites KX2-5036 $(n=14 / 18)$ and KX2-5163 $(n=13 / 18)$ were below the LLOQ of $0.05 \mathrm{ng} / \mathrm{mL}$.

Table 2. Tirbanibulin plasma PK parameters following 5 days of consecutive topical dosing

\begin{tabular}{|c|c|c|c|}
\hline Mean (SD) & & & \\
\hline $\begin{array}{l}\mathrm{C}_{\max }(\mathrm{ng} / \mathrm{mL}) \\
\mathrm{t}_{\text {max }}(\mathrm{h})\end{array}$ & $\begin{array}{l}0.340(0.297) \\
6.0(2.0,9.8)\end{array}$ & $\begin{array}{l}0.176(0.102) \\
7.8(2.0,10.0)\end{array}$ & $\begin{array}{c}0.258(0.231) \\
6.91(2.0,10.0)\end{array}$ \\
\hline $\mathrm{AUC}_{0.24}\left(\mathrm{~h}^{*} \mathrm{ng} / \mathrm{mL}\right)$ & $5.0(3.9)$ & $3.18(1.92)$ & $4.09(3.15)$ \\
\hline
\end{tabular}

- The mean (standard deviation, SD) age of subjects was 66.4 (9.42 [range: 43-83])

- Mean (SD) dose applied was 137 (44.9) mg among the combined subject group ( $55 \%$

Figure 1. (A) Mean trough plasma concentrations of tiban (B) Individual plasma concentrations of tirbanibulin with overall mean on Day 5 postdose

A

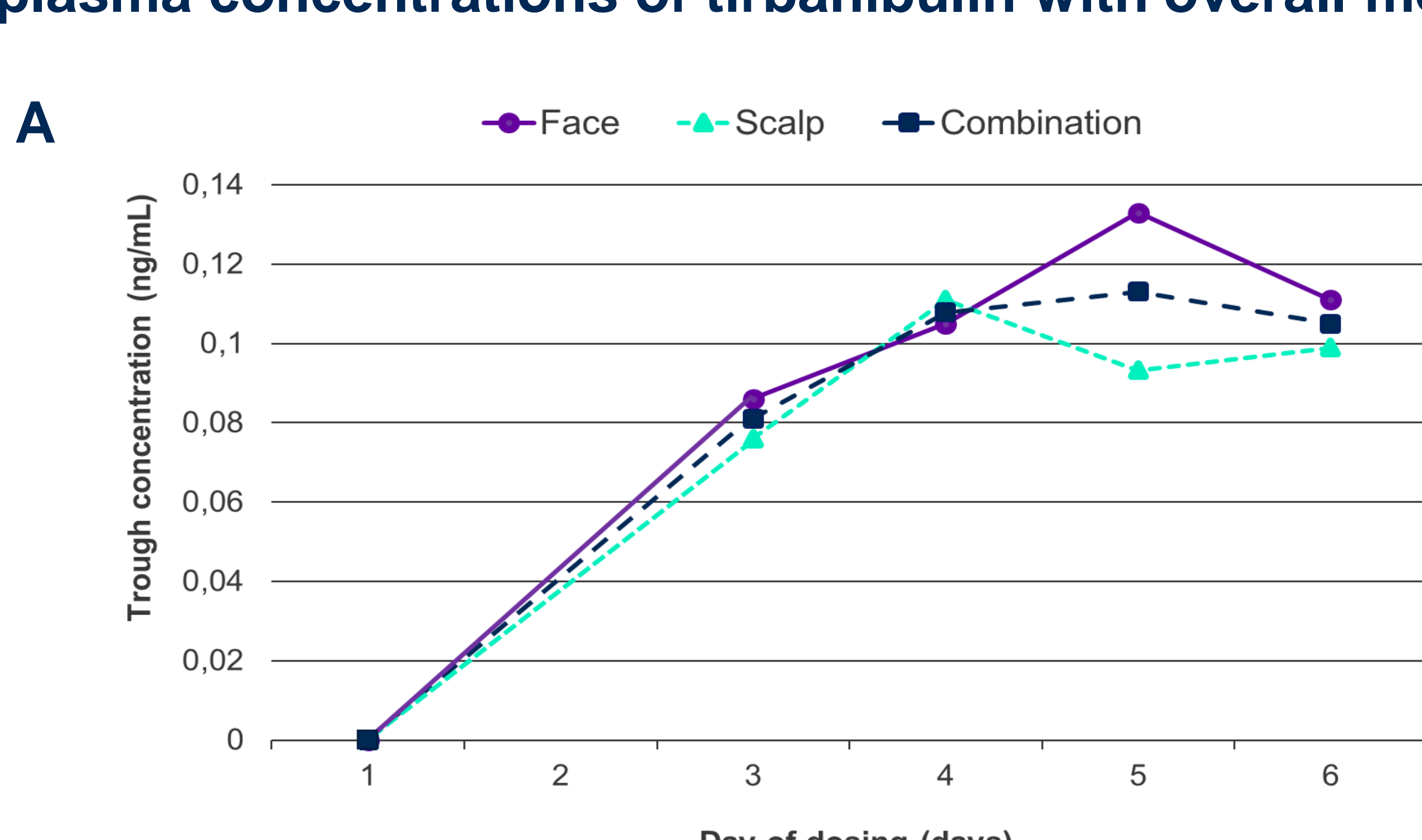

B

Day of dosing (days)

\section{Safety}

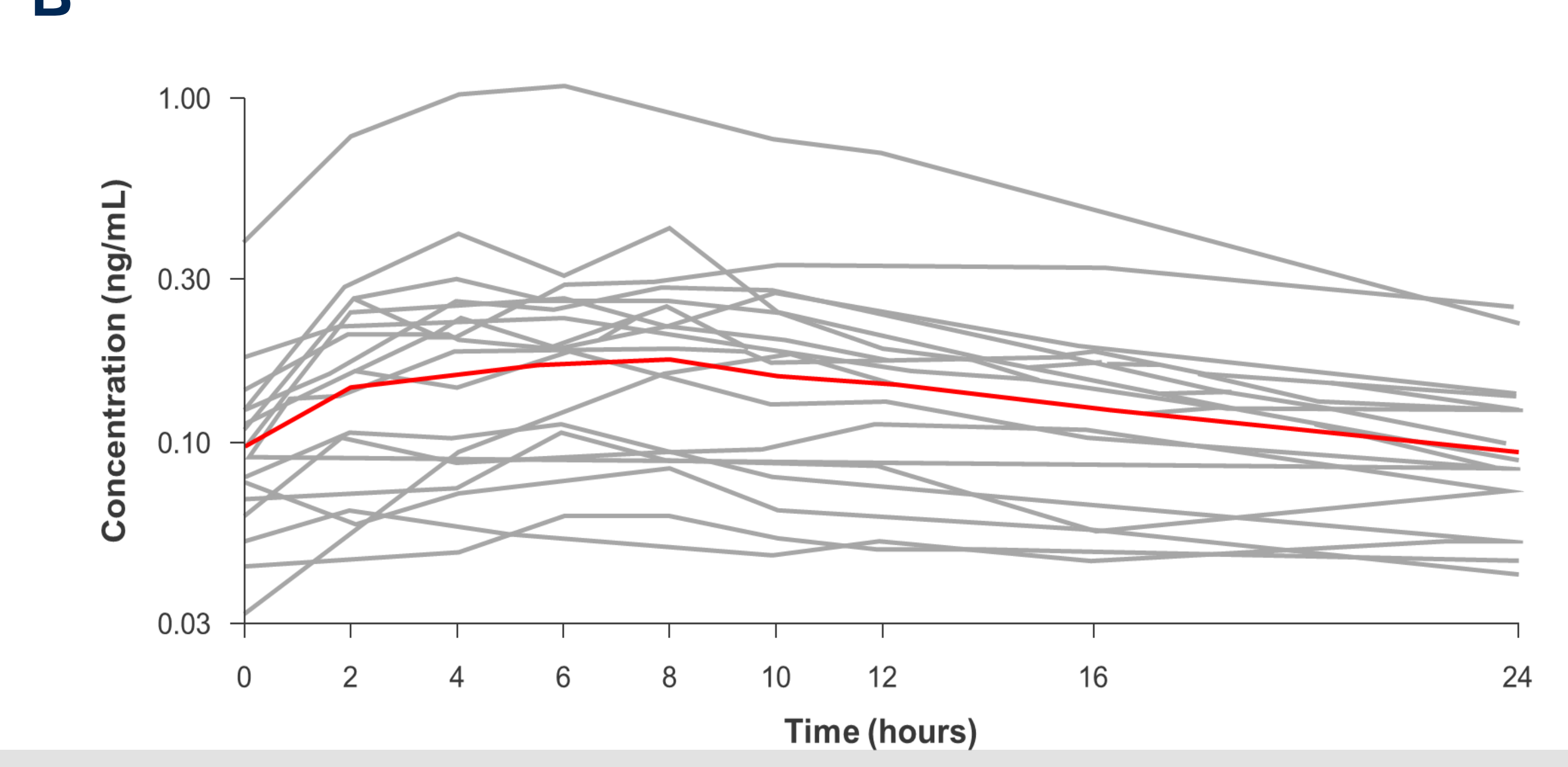

Adverse events

- Four subjects (face, $n=1$; scalp, $n=3$ ) experienced a total of 5 treatment-emergent AEs (TEAEs); all were unrelated to treatment.

- One subject in the scalp-treated group expenienced a treatment-related TEAE (mild skin dryness; resolved spontaneously.

- There were no serious AEs, severe AEs, deaths or TEAEs leading to study discontinuation.

Local skin reactions

LSRs on the treatment area were mostly transient, all were mild-to-moderate erythema and flaking/scaling that peaked around $D$

\section{CONCLUSIONS}

- Under maximal use conditions, low systemic exposure of tirbanibulin with subnanomolar plasma concentrations for both parent drug and metabolites was confirmed.

- Tirbanibulin ointment $1 \%$ for 5 days was well tolerated for the treatment of AK on the face/balding scalp.

REFERENCES 